\title{
Persistent olfactory complaints after COVID-19: a new interpretation of the psychophysical olfactory scores*
}

\author{
Clair Vandersteen', Magali Payne², Louise-Émilie Dumas², Victoria \\ Rhinology Online, Vol 4: 66 - 72, 2021 \\ Metelkina-Fernandez², Alexandra Plonka3,4, David Chirio5 , Élisa Demonchy5 , http://doi.org/10.4193/RHINOL/21.010 \\ Karine Risso ${ }^{5}$, Florence Askenazy-Gittard ${ }^{2,3}$, Nicolas Guevara1, Laurent \\ Castillo', Valeria Manera ${ }^{3}$, Auriane Gros ${ }^{1,3}$ \\ *Received for publication: \\ March 3, 2021 \\ 'Institut Universitaire de la Face et du Cou, Centre Hospitalier Universitaire, Université Côte d'Azur, Nice, Alpes-Maritimes, France Accepted: April 5, 2021 \\ 2 Hôpitaux Pédiatriques de Nice CHU-LENVAL, Centre Hospitalier Universitaire, Université Côte d'Azur, Nice, Alpes-Maritimes, \\ Published: April 26, 2021 \\ France \\ ${ }^{3}$ Laboratoire CoBTeK, Institut Claude Pompidou, Université Coté d'Azur, Nice, Alpes-Maritimes, France \\ ${ }^{4}$ Institut NeuroMod, INRIA Centre de recherche Sophia Antipolis, Université Côte d'Azur, Sophia Antipolis, Alpes-Maritimes, \\ France \\ ${ }_{5}^{5}$ Département de médecine infectiologique, hôpital de l'archet, Centre Hospitalier Universitaire, Université Côte d'Azur, Nice, \\ Alpes-Maritimes, France
}

\begin{abstract}
Background: Sudden olfactory loss is a major symptom of SARS-CoV-2 infection and has a negative impact on daily life quality. Almost $80 \%$ of disorders regress spontaneously. No precise characterization of the medium- and long-term olfactory symptoms has been carried out yet, apart from self-assessments. The main objective of this work was to characterize persistent smell disorders in this population.
\end{abstract}

Methodology: Consecutive patients consulting to the ENT department with post-Covid19 olfactory loss were included. The clinical examination included an analog scale for the self-assessment of olfactory recovery), a nasofibroscopy, the Sniffin' Stick Test and the short version of the Questionnaire of olfactory disorders.

Results: Among the 34 patients included, based on the Sniffin' Sticks Test, 29.4\% ( $n=10$ ) could be classified as normosmic, 55.9\% $(n=19)$ as hyposmic and $14.7 \%(n=5)$ as functional anosmic). Only olfactory identification impairment was significantly correlated with olfactory complaint and daily anxiety and annoyance related to lack of olfaction recovery. This identification disorder seemed to worsen over time.

Conclusions: It is crucial to assess odor identification disorders in case of persistent olfactory complaints after COVID-19. It is fundamental to target this disorder, as it does not improve spontaneously and negatively impact quality of life.

Key words: olfactory dysfunction, olfaction, sniffin sticks test, COVID-19, quality of life

\section{Introduction}

COVID-19 infection symptoms initially described were fever and cough in respectively 44 and $65 \%$ of cases $^{(1)}$. Currently, many studies report the frequency of anosmia preceding or during COVID19 seroconversion ${ }^{(2-5)}$. Anosmia can be the only COVID-19 symptom $^{(6)}$. So, sudden olfactory loss must lead to a COVID-19 screening ${ }^{(7)}$. Olfactory and taste loss are respectively reported, with great heterogenicity, in $25 \%$ to $98 \%$ and $15 \%$ to $85 \%$ of cases in COVID-19 patients ${ }^{(2,8,9)}$, especially because physicians used auto-questionnaires sent by e-mail, apps and mobiles to avoid obvious contagious situations. They are severe in $50 \%$ cases ( 22 to $80 \%$ of anosmia and $20 \%$ of ageusia ${ }^{(8,10)}$ ) and is more common in women over $50^{(2,3,11-13)}$. Psychophysical testing these acute COVID-19 patients allowed Lechien et al. ${ }^{(14)}$ to specify the extent of the acute olfactory loss to $80 \%$ of patients, $50 \%$ and $20 \%$ of them being respectively anosmic and hyposmic.

A patient with anosmia and loss of taste would be 6 times more likely to be infected with COVID-19 ${ }^{(8)}$, which in this specific case, 
would more frequently be a "mild" form that doesn't require hospitalization $^{(2)}$

The long-term anosmia can cause an alteration in the quality of life and psychiatric disorders such as depression ${ }^{(15,16)}$, anxiety, anorexia ${ }^{(17)}$ and its nutritional consequences ${ }^{(18)}$, social interaction disorders $^{(19,20)}$ and cognitive impairment ${ }^{(19,21,22)}$. So, the diagnosis of olfactory disorders and their management is essential. Spontaneous olfactory recovery between the first and third year after loss is observed in 32 to $66 \%{ }^{(23)}$ of other post-viral olfactory loss, not related to COVID-19 ${ }^{(23)}$. The early recovery rate (at 2 months) of post-viral olfactory loss post-COVID-19 is approximately $44 \%$ to $79 \%$ (of which $73 \%$ of patients recover within 8 days $)^{(2,23,24)}$. So, spontaneous olfactory recovery rate is better in of post- COVID-19 olfactory loss that in other post-viral olfactory loss such as Rhinovirus, Influenza, Respiratory syncytial virus or other Coronavirii. However, post- COVID-19 olfactory disorders appears to persist after 6 months in $60 \%{ }^{(25)}$ of patients (including $50 \%$ hyposmia and $10 \%$ anosmia with sometimes parosmia ${ }^{(26)}$ ). The principal aim of this study was to analyse the characteristics of persistent olfactory disorders post-COVID-19. The secondary aim was to measure their effects on olfaction-related quality of life.

\section{Materials and methods}

\section{Population}

The study was approved by the institutional review board of the Nice University Hospital (CNIL number: 412). This study is part of a large work registered under a ClinicalTrials.gov number (ID: NCT04799977). Since March 2020, we retroprospectively recruited at ENT department of Nice University Hospital all patients infected by COVID-19 with persistent olfactory disorders from two to nine months. Patients where self-referred or referred by colleagues, general practitioners or advised by the infectiology department that managed all COVID-19 declared patients (city guidelines). Patients had either an olfactory complaint for over 6 weeks and a molecular-proven SARS-CoV-2 diagnosis or a CT-proven SARS-CoV-2 diagnosis secondarily confirmed by serology. The patients' demographic data and clinical characteristics were recorded. Nasofibroscopy was performed to evaluate state and nasal cavity patency. The clinical examination included a visual analogue scale (VAS) for the subjective assessment of olfactory recovery (ranging from $0 \%$ to $100 \%$ ), an objective evaluation of olfactory loss using Sniffin'Sticks Test ${ }^{\circledR(27-29)}$ and the completion of a short version of Questionnaire of Olfactory Disorders (Short-QOD-NS) ${ }^{(30)}$.

\section{Nasofibroscopy}

Using a flexible endoscope and a high-definition camera, a nasofibroscopy was performed without local anesthesia (to avoid transient olfactive disorders) in order to assess the permeability of the olfactory cleft (presence of polyps, surgical adhesions, tumor or mucus was being sought).

\section{Objective olfactory disfunction}

Olfactory function was assessed using Sniffin' Sticks test, a validated psychophysical test that include an odor Threshold detection ( $T$ ), an odor Discrimination (D) and an odor Identification (I) tests ${ }^{(28,31-34)}$. During the test, subjects were blindfolded. Odor thresholds were determined for N-butanol (BUT), using a three-alternative forced choice task. Three sticks were presented to the patient in an alternating order, one containing the odorant, the other two containing solvents only. The subject's task was to find out which of the three pens smelled of the odorant. The odor discrimination test was performed using 16 triplets of odorants sticks. Subjects were presented with three sticks, two containing the same odorant, and one a different odorant. Through a forced choice, the patient's task was to identify the stick that smelled differently. For odor identification, 16 odorant sticks were presented once, separated by an interval of at least 20 seconds to prevent olfactory desensitization. Each stick presentation was accompanied by a written list containing the correct odorant and 3 semantic distractors. Results from the three tests, odor threshold ( $T$ ), odor discrimination (D), and odor identification (I) were summed up to a composite score, the socalled "TDI-score."

\section{Olfactory quality of life}

The olfactory quality of life was assessed using the Short-QODNS which is based on the Questionnaire of Olfactory Disorders (QOD) related to the consequences of an olfactory and taste loss such as the pleasure of sharing a meal, of creating social interactions or even of creating close bonds with others ${ }^{(35)}$. The original version was divided into 52 items regarding negative and positive social consequences of olfactory loss ${ }^{(36)}$. The "negative consequences" subdomain of QOD (QOD-NS $\left.{ }^{(37)}\right)$ has been shown to be more correlated with the results of psychophysical olfactory tests (Sniffin 'sticks tests) ${ }^{(38)}$. So, shorter versions have been developed to be more suitable for daily clinical practice $(30,39,40)$. These shorter versions increase the response rate and reduce the patient's mental load when completing the questionnaire ${ }^{(30)}$. The QOD-NS is a validated test ${ }^{(41)}$ which includes 17 questions with answers go from 0 to 3 for a total score of 0 to 51 (51 meaning there is no disorder). Finally, Mattos et al. ${ }^{(30)}$ developed an even shorter version (Short-QOD-NS) with the 7 most relevant questions with the different aspects such as social aspect $(n=3)$, eating $(n=2)$, anxiety $(n=1)$ and annoyance $(n=$ 1) following an olfactory loss. We have decided to use this version for this study, with score ranging from 0 to 21 (21 meaning there is no disorder).

Statistical analysis

Data are presented as mean (SD) for quantitative variables and 
Table 1. Demographic and clinical characteristics.

\begin{tabular}{|lcc|}
\hline & mean & SD \\
\hline Age (years) & 41.6 & 12.9 \\
\hline Months post-COVID-19 & 5.0 & 2.8 \\
\hline Total & n & $\%$ \\
\hline Sex & 34 & 100 \\
\hline Women & & \\
\hline Men & 16 & 47 \\
\hline COVID19 testing & 18 & 53 \\
\hline Molecular PCR test & & \\
\hline Chest CT & 24 & 82.8 \\
\hline Serology (antibody test) & 6 & 20.7 \\
\hline CoVID-19 dedicated treatment & 10 & 34.5 \\
\hline Oral corticosteroids & & \\
\hline Nasal corticosteroids & 5 & 14.6 \\
\hline Inhaled corticosteroids & 5 & 11.7 \\
\hline Azithromycin alone & 4 & 2.9 \\
\hline Azithromycin + Hydroxychloroquine & 4 & 11.7 \\
\hline Amoxicillin alone & 1 & 5.9 \\
\hline Amoxicillin + Azithromycin & 4.9 \\
\hline Others (vitamins, zinc) & 26.5 \\
\hline
\end{tabular}

$\mathrm{SD}=$ standard deviation; $\mathrm{CT}=$ computed tomography; $\mathrm{PCR}=$ polymerase chain reaction

as frequency and percentage for qualitative variables. In order to investigate correlations between subjective reports (VAS), objective disorders in the different dimension (threshold detection, $\mathrm{T}$; odor discrimination, D; odor identification I), and Short-QOD-NS, we performed bivariate correlation analyses. As data were not normally distributed (as suggested by Kolmogorov-Smirnov test), non-parametric Spearman's correlations were employed.

\section{Results}

Demographic and clinical features

Thirty-four patients consulting the ENT department of Nice University Hospitals (CHU) for olfactory complaints after a COVID-19 infection were included in the study. The demographic and clinical features are reported in Table $1.47 \%$ of whom were female $(n=16)$, with a mean age of $41 \pm 12$ years. They were seen after $5 \pm 2,8$ months after the COVID-19 infection. Twentyeight patients received a COVID-19 related treatment.

\section{Loss of smell and taste}

Descriptive analyses for the loss of smell and taste are reported in Table 2. The day of consultation, patients reported to have recovered $37 \pm 27 \%$ of their olfaction (ranging from $0 \%$ to $90 \%$ ). The global results of the Sniffin' Sticks Test (TDI) suggested that
Table 2. Smell and taste disorders, olfactory quality of life.

\begin{tabular}{|lcc|}
\hline & mean & SD \\
\hline VAS (subjective \% of recuperation) & 37.2 & 26.5 \\
\hline Short-QOD-NS - Total & 11.1 & 5.0 \\
\hline Short-QOD-NS-Eating & 3.1 & 2.2 \\
\hline Short-QOD-NS-Anxiety & 2.0 & 1.0 \\
\hline Short-QOD-NS-Annoyance & 1.2 & 1.2 \\
\hline Short-QOD-NS-Social & 4.8 & 2.6 \\
\hline Sniffin' Sticks test - scores & & \\
\hline Threshold detection & 5.4 & 4.0 \\
\hline Discrimination & 10.9 & 2.6 \\
\hline Identification & 10.2 & 2.7 \\
\hline & $\mathbf{n}$ & $\%$ \\
\hline Sniffin' Sticks test - classification & & \\
\hline Normosmic & 10 & 29.4 \\
\hline Hyposmic & 19 & 55.9 \\
\hline Anosmic & 5 & 14.7 \\
\hline Taste disorders & 30 & 88.2 \\
\hline Retro-olfaction alone & 22 & 73.4 \\
\hline Retro-olfaction + taste & 7 & 23.3 \\
\hline Taste alone & 5.3 \\
\hline
\end{tabular}

Short-QOD-NS =Short version of Questionnaire of Olfactory Disorders(30)

$29,4 \%(n=10)$ of the patients could be classified as normosmic (TDI $\geq 30.75), 55,9 \%(n=19)$ as hyposmic ( $16.25 \leq$ TDI $\leq 30.5)$ and $14,7 \%(n=5)$ as anosmic (TDI $\leq 16)$. Eighty eight percent of the patients $(n=30)$ reported taste disorders, including retro-olfaction (food flavors) alone $(73,4 \%, n=22)$, retro-olfaction associated to taste $(23,3 \%, n=7,66 \%$ concerning sweet and salty, $33 \%$ concerning sour and bitter), or taste alone $(3,3 \%, \mathrm{n}=1$ concerning sweet and salty).

Links between self-reported and objective olfactory disorders Correlations between subjective reports (VAS) and the T, D and I scores of the Sniffin' Stick Test suggested a significant, positive correlation between percentage of subjective olfactory recovery (VAS) and odor identification $\left(\mathrm{I}, \mathrm{rho}_{(32)}=0.36, \mathrm{p}=0.034\right)$. Correlations with odor threshold detection and discrimination were not-significant $\left(T, r h o(32)=0.23, p=0.193 ; \mathrm{D}, \mathrm{rho}_{(32)}=0.23, \mathrm{p}=\right.$ 0.184). Correlations between the three Sniffin' Sticks Test subscales and the months after the first COVID-19 infection were not statistically significant. However, while objective disorders in odor detection seemed not to improve over time $\left(\mathrm{rho}_{(32)}=0.20\right.$, $p=0.260$ ), disorders in odor identification showed an alternative pattern. A longer timeframe from the first infection was associated a non-significant trend towards decrease of identification 


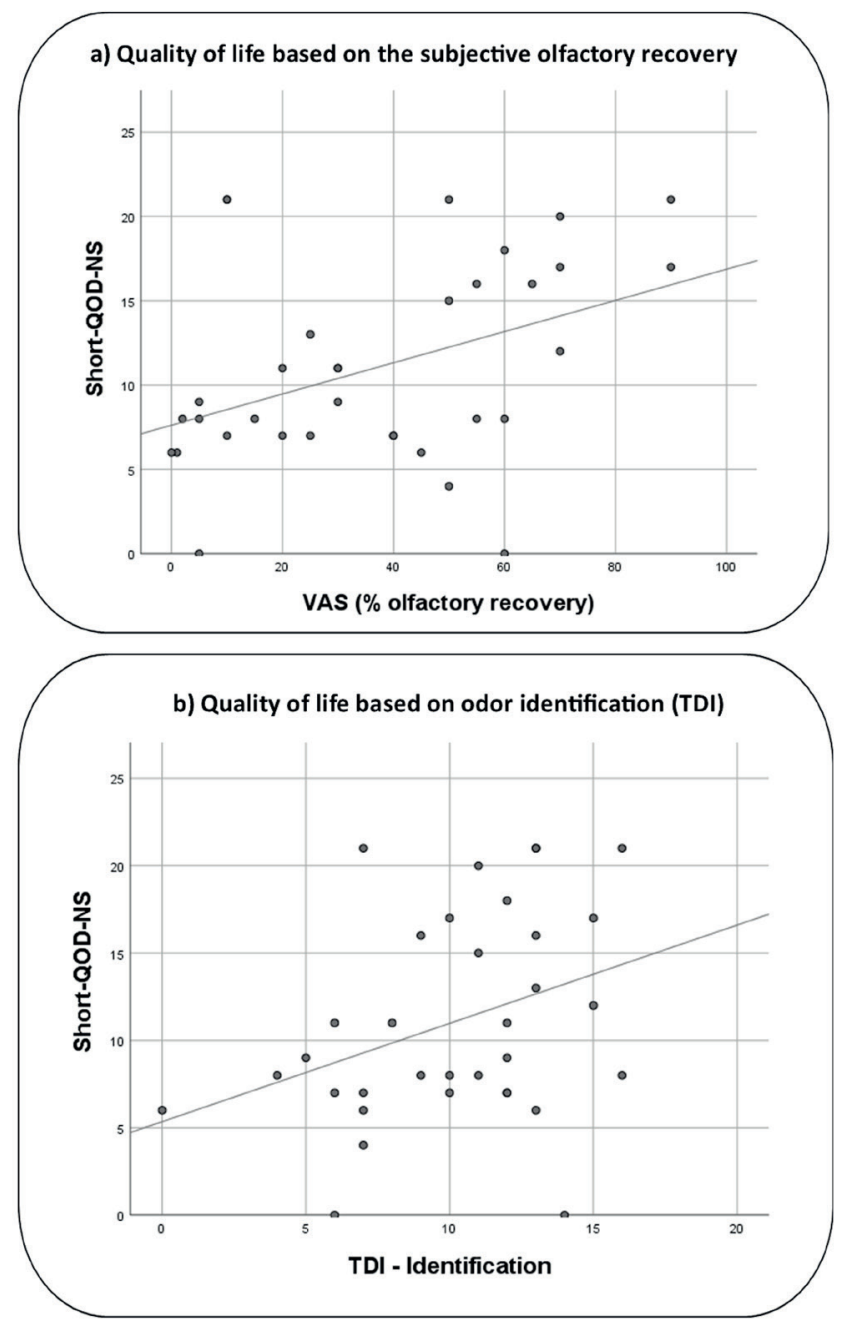

Figure 1. Correlations between quality of life (Short-QOD-NS) and percentage of subjective odor recovery (VAS; a), and objective odor identification scores (TDI-I; b).

performance $\left(\mathrm{rho}_{(32)}=-0.24, \mathrm{p}=0.177\right)$.

Links between olfactory disorders and quality of life Descriptive analyses of the Short-QOD-NS are reported in Table 2. Spearman's correlations revealed that the global ShortQOD-NS was positively correlated to self-reported percentage of olfactory recovery $\left(\mathrm{VAS}, \mathrm{rho}_{(32)}=0.42, \mathrm{p}=0.014\right.$, see Figure 1a). When considering the different Short-QOD-NS subscales, the only significant correlation was found for the Short-QODNS-eating $\left(\mathrm{rho}_{(32)}=0.45, \mathrm{p}=0.008\right)$. Concerning correlations between Short-QOD-NS and the Sniffin' Sticks Test scores, odor identification was positively correlated with the global ShortQOD-NS scale $\left(\right.$ rho $_{(32)}=0.36, p=0.037$, see Figure $\left.1 b\right)$. Specifically, significant correlations were found for the Short-QOD-NS-anxiety $\left(\mathrm{rho}_{(32)}=0.39, \mathrm{p}=0.025\right)$ and the Short-QOD-NS-Annoyance question $\left(\mathrm{rho}_{(32)}=0.37, \mathrm{p}=0.031\right)$. No significant correlation was found between Short-QOD-NS (global score and subscales) and the detection and discrimination scores (all ps $>0.13$ ).

\section{Discussion}

This is the first study that evaluates quantitatively olfactory disorders and their influence on the quality of life of 34 patients after five months on average from their olfactory loss due to a SARS-CoV-2 infection. This study is also the first to show a mismatch between subjective olfactory complaints of postCOVID-19 patients and the results of objective psychophysical tests. In fact, half of the patients had a persistent olfactory complaint whereas, based on the Sniffin Test, only 15\% ( $n=5)$ of them were classified as functional anosmic. In the same way, $30 \%$ of normosmic at Sniffin' Stick test (TDI) patients reported only partial recovery of their previous olfaction (VAS $=40 \%[5 \%$ $-70 \%]$ ). A previous study showed that $6 \%$ of patients infected with COVID-19 who presented an olfactory complaint after 12 days from onset of symptoms were normosmic on the Sniffin' Stick test ${ }^{(14)}$.

Subdomain Sniffin' Stick test analyses employing recent normative data ${ }^{(33)}$ highlights a predominant disorder on the identification (I) of the odorant, which is more important than the odor discrimination (D) or the detection threshold $(\mathrm{T})$. Also, normosmic patients $(\mathrm{TDI}>30.75)^{(33)}$ had more altered scores in odor identification (I) than in detection $(T)$. The results of this study raise the question of an unrecognized central involvement of olfaction, compatible with an olfactory agnosia type ${ }^{(34,42)}$. Indeed, Whitcroft et al. ${ }^{(42)}$ have shown that the impairment of odor identification compared to the impairment of threshold detection, was more frequently found in central damage sequelae of neuronal lesions of infectious, traumatic or degenerative origin. On the contrary, it has been shown that the isolated impairment of detection is mainly the consequence of sinonasal pathologies ${ }^{(42)}$. These sinonasal symptoms ${ }^{(43,44)}$ reduce olfactory perception (obstruction of the olfactory cleft, mucous edema) but decrease significantly only the olfactory threshold and not the identification, as we observed in our study. This observation highlights the independence of the central processing of the olfactory message in relation to the peripheral perception of the odorant at the level of the olfactory epithelium. Our results are in line with previous studies, which hypothesized a central impairment of olfaction due to contact of the membrane viral glycoprotein S1 with ACE2 facilitated by TMPRSS2 ${ }^{(45-48)}$. Indeed, more and more reports suggest the penetration of SARS-CoV-2 into the central nervous system ${ }^{(49,50)}$ through the olfactory cleft through the olfactory epithelium, and more particularly through sustentacular and / or trans-cribriform sheath cells ${ }^{(47,51,52)}$, through a rupture of the blood-brain barrier ${ }^{(53,54)}$ or by trans-axonal feedback ${ }^{(50,55)}$ within the peripheral nerves coming from the respiratory tree (Vagus nerve). Finally, previous SARS-CoV-1 studies results ${ }^{(56)}$ and the olfactory identification impairment found in neurodegenerative diseases, raised questions about the pathophysiological similarities and consequences of SARS-CoV-2 cells and the mechanisms involved in the origin of neurodegenerative 
diseases (57).

This study did not show any significant correlation between the olfactory disorder duration and the severity of the olfactory impairment. However, we observed a non-significative tendency for identification disorders to increase over time, rather than to reduce. This pattern has to be confirmed using longitudinal data. If this observation will be confirmed by other authors, two hypotheses can be put forward. The first is an early progressive deafferentation ${ }^{(58)}$ of central olfactory projections, which negatively influences the cognitive performance of these patients. In this way, Lu et al. ${ }^{(59)}$ found an alteration in cerebral trophicity on MRI at the level of the olfactory cortex, the hippocampus, the Insula, the left Rolandic operculum, the Heschl gyrus, left and right cingulate about 3 months from the end of COVID-19 symptoms. The second is the emergence of new variants of SARS-CoV-2 in which the Spike S1 protein is said to have an increasing affinity in vivo with ACE2. This physiopathological mechanism described by Butowt et al. ${ }^{(60,61)}$ is thought to have its origin in a mutation of an aspartic acid to glycine at position 614 of the code for the Spike S1 protein (D614G). This mutation, associated with a genetic and geographic polymorphism of ACE2 ${ }^{(62)}$, could potentially cause an increase in the prevalence of olfactory damage, specifically in Europe ${ }^{(61)}$.

In addition, this study shows an isolated taste disorders (sweet, salty, sour, bitter and umami) in $3.3 \%$ of cases. This is in line with the results of Hopkins et al. ${ }^{(25)}$, that found $2.8 \%$ of persistent taste disturbances 6 months after COVID-19 infection. Taste loss can be explained by the tantalizing effect of ACE2 involvement on the taste buds ${ }^{(63)}$, which remains the main receptor targeted by SARS-CoV-2. However, the intricacy of primary taste and retrognathic olfaction in patient complaints makes its interpretation difficult. Taste loss disappears mainly within the first 15 days after the onset of symptoms, in particular thanks to the fast turnover of taste buds in the average time of 10 days ${ }^{(63)}$. Their persistence beyond 10 days suggests a possible role of the retrognathic olfaction in the perception of a taste disorders by the patients, which confirms the significant impact of an olfactory loss on the quality of food life showed in this study.

Finally, this study highlights the need of further studies on central consequences of post-COVID-19 olfactory loss, especially regarding impaired semantic memory of odours in patients with persistent olfactory complaints. Moreover, these patients, considered to be normosmic in olfactory tests but nevertheless symptomatic, underline the limits of psychophysical tests and suggest the need to adapt them, specially concerning the central processing disorders of olfactory diagnosis.

Olfaction assessment is most important as olfaction impairments can be the cause of domestic accidents (gas, burning smell) ${ }^{(17)}$, can testify the emergence of psychological(19), psychiatric $^{(15,16,19)}$, or eating disorders ${ }^{(64,65)}$, as well as loss of taste pleasure ${ }^{(66)}$ and social isolation for fear of one's own body odor, or even eating spoiled or undercooked food.

Smell loss will therefore cause a significant deterioration in the quality of life ${ }^{(35,39,67)}$, especially since the loss occurs before the age of $30^{(67)}$. In ENT studies, 22-item Sinonasal Outcome Test (SNOT-22) $)^{(68)}$ is commonly used for quality-of-life assessment but is not focused on smell and taste impairment. QOD and specifically QOD-NS ${ }^{(37,40,67,69)}$ is rather used for this specific olfaction assessment. However, QOD-NS 17-item questionnaire length can be a problem in clinical and scientific research as patients' mental burden can be important. In order to improve efficiency and the quality of collected data, Mattos et al. ${ }^{(30)}$ suggested to use a Short-QOD-NS, reporting an strong correlation with QODNS total and domain-specific scores. Even if it is not internationally validated, we have chosen this quality-of-life assessment because it fit betters with the constraints of the routine clinical assessment. In our study, Short-QOD-NS results allowed us to underline a negative correlation between olfactory loss and quality of life, and more particularly on the pleasure of eating, which is often the predominant patient's complaint. Specifically, an odor identification disorder generates anxiety and concern for patients regarding the lack of smell recovery. In a previous study, Lechien et al. ${ }^{(62)}$ already showed, at about 10 days of the onset of symptoms of COVID-19, a degradation of Short-QODNS, which is more significant in anosmic patients and predominant in their daily worry about habituation to this disorder. Their results showed a deterioration in the overall quality of an olfactory life with the duration of olfactory deprivation ${ }^{(62)}$. Despite these interesting results, this study suffers from some limitations. The main limitation concerns the small cohort of 34 patients, with no follow up reported, who spontaneously consulted our university hospital, which represents the risk of a recruitment bias. The small sample size may have contributed to a limited strength of correlations $\left(\mathrm{rho}_{(32)} \mathrm{MAX}=0,45\right)$, and therefore our results cannot be directly generalized to all patients with a post-covid olfactory disorder.

\section{Conclusion}

This work analyzes long term olfactory disorders occurring after COVID-19 infection. Sniffin' Stick Tests results are mismatching with self-reported complains in olfactory loss. Indeed, the Sniffin' Stick Tests evaluated $30 \%$ of subjectively affected patients as normosmic. We highlighted the presence of a significant impairment in odor identification that should be targeted by a specific olfactory training added to these patients' care, especially because it significantly worsens quality of life and does not seem to spontaneously recover over time.

\section{Authorship contribution}

CV, MP, LED, VMF, AG contributed to study design, data collection, interpretation of results, drafting and critical evaluation of the final manuscript. AP, DC, ED, KR, NG and LC contributed 
to study design, interpretation of results, drafting and critical evaluation of the final manuscript. VM contributed to biostatistics, interpretation of results and critical evaluation of the final manuscript.

\section{Acknowledgments}

Not applicable.

\section{Funding}

Not applicable.

\section{Ethics approval and consent to participate}

Not applicable.

\section{Consent for publication}

Not applicable.

\author{
Availability of data and materials \\ Not applicable.
}

Conflict of interest

The authors declare no conflict of interest.

\section{References}

1. Guan W, Ni Z, Hu Y, et al. Clinical Characteristics of Coronavirus Disease 2019 in China. N Engl J Med. 2020;382(18):170820.

2. Costa KVT da, Carnaúba ATL, Rocha KW, Andrade KCL de, Ferreira SMS, Menezes $P$ de L. Olfactory and taste disorders in COVID-19: a systematic review. Braz J Otorhinolaryngol. 2020;86(6):781-792.

3. Dawson P, Rabold EM, Laws RL, et al. Loss of Taste and Smell as Distinguishing Symptoms of Coronavirus Disease 2019 Clin Infect Dis. 2021;72(4):682-5.

4. Renaud M, Leon A, Trau G, et al. Acute smell and taste loss in outpatients: all infected with SARS-CoV-2? Rhinology. 2020;58(4):406-409.

5. Heidari F, Karimi E, Firouzifar $M$, et al. Anosmia as a Prominent Symptom of COVID-19 Infection. Rhinology. 2020;58(3):302-3.

6. Gane SB, Kelly C, Hopkins C. Isolated Sudden Onset Anosmia in COVID-19 Infection. A Novel Syndrome? Rhinology. 2020;58(3):299-301.

7. Saussez S, Lechien JR, Hopkins C. Anosmia: an evolution of our understanding of its importance in COVID-19 and what questions remain to be answered. Eur Arch OtoRhino-Laryngology. 2020;0-4

8. Carrillo-Larco RM, Altez-Fernandez C. Anosmia and dysgeusia in COVID-19: A systematic review. Wellcome Open Res. 2020:5:94.

9. Borsetto D, Hopkins C, Philips V, et al. Self-reported alteration of sense of smell or taste in patients with COVID-19: a systematic review and meta-analysis on 3563 patients. Rhinology. 2020;58(5):430-436.

10. Lechien JR, Chiesa-Estomba CM, Place S, et al. Clinical and epidemiological characteristics of 1420 European patients with mild-tomoderate coronavirus disease 2019. J Intern Med. 2020;288(3):335-44.

11. Samaranayake LP, Fakhruddin KS, Panduwawala C. Sudden onset, acute loss of taste and smell in coronavirus disease 2019 (COVID-19): a systematic review. Acta Odontol Scand. 2020;78(6):467-73.

12. Agyeman $A A$, Chin $\mathrm{KL}$, Landersdorfer $C B$
Liew D, Ofori-Asenso R. Smell and Taste Dysfunction in Patients With COVID-19: A Systematic Review and Meta-analysis. Mayo Clin Proc. 2020;95(8):1621-31.

13. Boscolo-Rizzo P, Polesel J, Spinato G, et al Predominance of an altered sense of smel or taste among long-lasting symptoms in patients with mildly symptomatic COVID19. Rhinology. 2020;58(5):524-5

14. Lechien JR, Cabaraux P, Chiesa-Estomba CM, et al. Psychophysical Olfactory Tests and Detection of COVID-19 in Patients With Sudden Onset Olfactory Dysfunction: A Prospective Study. Ear, Nose Throat J. 2020;99(9):579-83.

15. Hur K, Choi JS, Zheng M, Shen J, Wrobel B. Association of alterations in smell and taste with depression in older adults. Laryngoscope Investig Otolaryngol. 2018;3(2):94-9.

16. Kohli P, Soler ZM, Nguyen SA, Muus JS Schlosser RJ. The Association Between Olfaction and Depression: A Systematic Review. Chem Senses. 2016:41(6):479-86.

17. Croy I, Nordin S, Hummel T. Olfactory disorders and quality of life-an updated review. Chem Senses. 2014;39(3):185-94.

18. Nordin S. Sensory perception of food and ageing. In: Food for the Ageing Population. Elsevier; 2009. p. 73-94.

19. Valsamidis K, Printza A, Constantinidis J, Triaridis S. The Impact of Olfactory Dysfunction on the Psychological Status and Quality of Life of Patients with Nasal Obstruction and Septal Deviation. Int Arch Otorhinolaryngol. 2020;24(02):e237-46.

20. Schablitzky S, Pause BM. Sadness might isolate you in a non-smelling world: olfactory perception and depression. Front Psychol. 2014;5(FEB).

21. Nordin S, Brämerson A. Complaints of olfactory disorders: epidemiology, assessment and clinical implications. Curr Opin Allergy Clin Immunol. 2008;8(1):10-5.

22. Ahmedy F, Mazlan M, Danaee M, Abu Bakar MZ. Post-traumatic brain injury olfactory dysfunction: factors influencing quality of life. Eur Arch Oto-Rhino-Laryngology. 2020;277(5):1343-51.

23. Kanjanaumporn J, Aeumjaturapat S, Snidvongs K, Seresirikachorn K, Chusakul
S. Smell and taste dysfunction in patients with SARS-CoV-2 infection: A review of epidemiology, pathogenesis, prognosis, and treatment options. Asian Pacific J Allergy Immunol. 2020;69-77.

24. Speth MM, Singer-Cornelius T, Oberle $M$, Gengler I, Brockmeier SJ, Sedaghat AR. Time scale for resolution of olfactory dysfunction in COVID-19. Rhinology. 2020;58(4):404-405

25. Hopkins C, Surda P, Vaira LA, et al. Six month follow-up of self-reported loss of smell during the COVID-19 pandemic. Rhinology. 2021;59(1):26-31.

26. Cook E, Kelly C, Watson DB, Hopkins C. Parosmia is prevalent and persistent amongst those with COVID-19 olfactory dysfunction. Rhinology. 2021;59(2):222-224.

27. Allis TJ, Leopold DA. Smell and Taste Disorders. Facial Plast Surg Clin North Am. 2012:20(1):93-111.

28. Hummel T, Sekinger B, Wolf SR, Pauli E, Kobal G. 'Sniffin' Sticks': Olfactory Performance Assessed by the Combined Testing of Odour Identification, Odor Discrimination and Olfactory Threshold. Chem Senses. 1997;22(1):39-52.

29. Rumeau C, Nguyen DT, Jankowski R. Comment tester l'olfaction avec le Sniffin' Sticks test ${ }^{\circledR}$. Ann françaises d'Oto-rhino-laryngologie Pathol Cervico-faciale. 2016;133(3):183-6.

30. Mattos JL, Edwards C, Schlosser RJ, et al. A brief version of the questionnaire of olfactory disorders in patients with chronic rhinosinusitis. Int Forum Allergy Rhinol. 2019;9(10):1144-50.

31. Gudziol V, Lötsch J, Hähner A, Zahnert T, Hummel T. Clinical significance of results from olfactory testing. Laryngoscope. 2006;116(10):1858-63.

32. Steinbach S, Hummel T, Böhner C, et al. Qualitative and Quantitative Assessment of Taste and Smell Changes in Patients Undergoing Chemotherapy for Breast Cancer or Gynecologic Malignancies. J Clin Oncol. 2009;27(11):1899-905.

33. Oleszkiewicz A, Schriever VA, Croy I, Hähner A, Hummel T. Updated Sniffin' Sticks normative data based on an extended sample of 9139 subjects. Eur Arch Oto-RhinoLaryngology. 2019;276(3):719-28. 
34. Hummel T, Whitcroft KL, Andrews $P$, et al Position paper on olfactory dysfunction. Rhinology. 2017;54(26):1-30.

35. Brämerson A, Nordin S, Bende M. Clinica experience with patients with olfactory complaints, and their quality of life. Acta Otolaryngol. 2007;127(2):167-74.

36. Frasnelli J, Hummel T. Olfactory dysfunction and daily life. Eur Arch Oto-RhinoLaryngology. 2005;262(3):231-5

37. Mattos JL, Schlosser RJ, DeConde AS, et al. Factor analysis of the questionnaire of olfactory disorders in patients with chronic rhinosinusitis. Int Forum Allergy Rhinol. 2018;8(7):777-82

38. Simopoulos $E$, Katotomichelakis $M$, Gouveris H, Tripsianis G, Livaditis M Danielides V. Olfaction-associated quality of life in chronic rhinosinusitis: Adaptation and validation of an olfaction-specific questionnaire. Laryngoscope. 2012;122(7):1450-4.

39. Smeets MAM, Veldhuizen MG, Galle $S$, et al. Sense of Smell Disorder and HealthRelated Quality of Life. Rehabil Psychol. 2009:54(4):404-12.

40. Soler ZM, Smith TL, Alt JA, Ramakrishnan VR, Mace JC, Schlosser RJ. Olfactory-specific quality of life outcomes after endoscopic sinus surgery. Int Forum Allergy Rhinol. 2016;6(4):407-13.

41. Rudmik L, Hopkins C, Peters A, Smith TL, Schlosser RJ, Soler ZM. Patient-reported outcome measures for adult chronic rhinosinusitis: A systematic review and quality assessment. J Allergy Clin Immunol. 2015;136(6):1532-1540.e2

42. Whitcroft $\mathrm{KL}$, Cuevas M, Haehner A Hummel T. Patterns of olfactory impairment reflect underlying disease etiology. Laryngoscope. 2017;127(2):291-5.

43. Åkerlund A, Bende M, Murphy C. Olfactory Threshold and Nasal Mucosal Changes in Experimentally Induced Common Cold Acta Otolaryngol. 1995;115(1):88-92.

44. Stuck BA, Hummel T. Olfaction in allergic rhinitis: A systematic review. J Allergy Clin Immunol. 2015;136(6):1460-70.

45. Bilinska K, Butowt R. Anosmia in COVID19: A Bumpy Road to Establishing a Cellular Mechanism. ACS Chem Neurosci. 2020;11(15):2152-5.

46. Fodoulian L, Tuberosa J, Rossier D, et al SARS-CoV-2 Receptors and Entry Genes Are Expressed in the Human Olfactory Neuroepithelium and Brain. iScience. 2020;23(12):101839.

47. Butowt R, Bilinska K. SARS-CoV-2: Olfaction, Brain Infection, and the Urgent Need for Clinical Samples Allowing Earlier Virus Detection. ACS Chem Neurosci. 2020;11(9):1200-3.

48. Ueha R, Kondo K, Kagoya R, Shichino S, Ueha S, Yamasoba T. ACE2, TMPRSS2, and Furin expression in the nose and olfac- tory bulb in mice and human. Rhinology. 2021;59(1):105-109.

49. Meinhardt J, Radke J, Dittmayer C, et al. Olfactory transmucosal SARS-CoV-2 invasion as a port of central nervous system entry in individuals with COVID-19. Nat Neurosci. 2021;24(2):168-75.

50. Baig AM, Khaleeq A, Ali U, Syeda $H$. Evidence of the COVID-19 Virus Targeting the CNS: Tissue Distribution, Host-Virus Interaction, and Proposed Neurotropic Mechanisms. ACS Chem Neurosci. 2020;11(7):995-8.

51. Fodoulian L, Tuberosa J, Rossier D, et al. SARS-CoV-2 Receptors and Entry Genes Are Expressed in the Human Olfactory Neuroepithelium and Brain. iScience. 2020;23(12):101839.

52. Brann DH, Tsukahara T, Weinreb C, et al. Non-neuronal expression of SARSCoV-2 entry genes in the olfactory system suggests mechanisms underlying COVID-19-associated anosmia. Sci Adv. 2020;6(31):eabc5801.

53. Lee M-H, Perl DP, Nair G, et al. Microvascular Injury in the Brains of Patients with Covid19. N Engl J Med. 2021;384(5):481-3.

54. Paniz-Mondolfi A, Bryce C, Grimes Z, et al. Central nervous system involvement by severe acute respiratory syndrome coronavirus-2 (SARS-CoV-2). J Med Virol. 2020;92(7):699-702

55. Toljan K. Letter to the Editor Regarding the Viewpoint "Evidence of the COVID-19 Virus Targeting the CNS: Tissue Distribution, Host-Virus Interaction, and Proposed Neurotropic Mechanism". ACS Chem Neurosci. 2020;11(8):1192-4

56. Mahalaxmi I, Kaavya J, Mohana Devi S, Balachandar V. COVID-19 and olfactory dysfunction: A possible associative approach towards neurodegenerative diseases. J Cell Physiol. 2021;236(2):763-70

57. Singh AK, Bhushan B, Maurya A, Mishra G, Singh SK, Awasthi R. Novel coronavirus disease 2019 (COVID-19) and neurodegenerative disorders. Dermatol Ther. 2020;33(4) e13591.

58. Karimi-Galougahi M, Yousefi-Koma $A$, Bakhshayeshkaram M, Raad N, Haseli S. 18FDG PET/CT Scan Reveals Hypoactive Orbitofrontal Cortex in Anosmia of COVID19. Acad Radiol. 2020;27(7):1042-3.

59. Lu Y, Li X, Geng D, et al. Cerebral MicroStructural Changes in COVID-19 Patients - An MRI-based 3-month Follow-up Study. EClinicalMedicine. 2020;25(2):100484.

60. Butowt R, Bilinska K, Von Bartheld CS Chemosensory Dysfunction in COVID-19: Integration of Genetic and Epidemiological Data Points to D614G Spike Protein Variant as a Contributing Factor. ACS Chem Neurosci. 2020;11(20):3180-4.

61. von Bartheld CS, Hagen MM, Butowt R
Prevalence of Chemosensory Dysfunction in COVID-19 Patients: A Systematic Review and Meta-analysis Reveals Significant Ethnic Differences. ACS Chem Neurosci. 2020;11(19):2944-61.

62. Lechien JR, Chiesa-Estomba CM, De Siat DR, et al. Olfactory and gustatory dysfunctions as a clinical presentation of mild-tomoderate forms of the coronavirus disease (COVID-19): a multicenter European study. Eur Arch Oto-Rhino-Laryngology. 2020;277(8):2251-61.

63. Vaira LA, Deiana G, Fois AG, et al. Objective evaluation of anosmia and ageusia in COVID-19 patients: Single-center experience on 72 cases. Head Neck. 2020;42(6):1252-8.

64. Roessner V, Bleich S, Banaschewski T, Rothenberger A. Olfactory deficits in anorexia nervosa. Eur Arch Psychiatry Clin Neurosci. 2005;255(1):6-9.

65. Islam MA, Fagundo AB, Arcelus J, et al. Olfaction in eating disorders and abnormal eating behavior: a systematic review. Front Psychol. 2015;6.

66. Liu DT, Besser G, Prem B, et al. Selfperceived Taste and Flavor Perception: Associations With Quality of Life in Patients With Olfactory Loss. Otolaryngol Neck Surg. 2020;019459982096524

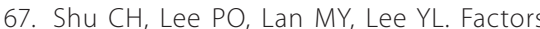
Affecting the Impact of Olfactory Loss on the Quality of Life and Emotional Coping Ability. Rhinol J. 2011;49(3):337-41

68. Hopkins C, Gillett S, Slack R, Lund VJ, Browne JP. Psychometric validity of the 22-item Sinonasal Outcome Test. Clin Otolaryngol. 2009;34(5):447-54.

69. Soler ZM, Hyer JM, Karnezis TT, Schlosser RJ The Olfactory Cleft Endoscopy Scale correlates with olfactory metrics in patients with chronic rhinosinusitis. Int Forum Allergy Rhinol. 2016;6(3):293-8.

\section{Dr. Clair Vandersteen, MD}

ENT surgery departement of Institut Universitaire de la Face et du Cou

(IUFC)

31 Avenue de Valombrose

Centre Hospitalier Universitaire (CHU)

Université Côte D’Azur (UCA)

06100 Nice

France

Tel : +33-(0)4-9203 1761

Fax : +33-(0)4-9203 1705

E-mail : vandersteen.c@chu-nice.fr ORCID: 0000-0001-5597-2

ISSN: 2589-5613 / @2021 The Author(s). This work is licensed under a Creative Commons Attribution 4.0 International License. The images or other third party material in this article are included in the article's Creative Commons license, unless indicated otherwise in the credit line; if the material is not included under the Creative Commons license, users will need to obtain permission from the license holder to reproduce the material. To view a copy of this license, visit http://creativecommons.org/ licenses/by/4.0/ 